\title{
A Brief Overview of the Complex Biological and Engineering Networks
}

\author{
Jinhu Lü \\ Key Laboratory of Systems and Control, Institute \\ of Systems Science, Academy of Mathematics and \\ Systems Science, Chinese Academy of Sciences \\ Beijing 100080, P. R. China \\ Email: jhlu@iss.ac.cn
}

\author{
Derong Liu \\ Department of Electrical and Computer Engineering \\ University of Illinois at Chicago \\ Chicago, IL 60607-7053, USA \\ Email: dliu@ece.uic.edu
}

\begin{abstract}
Over the last few decades, complex networks have been intensively studied throughout many fields of science, especially in biological and engineering sciences. This paper briefly reviews the main advances in the complex biological and engineering networks, aiming to bridge the gap between the complex biological and engineering networks. Biologists pay more attention to the mechanisms and local dynamics of individuals, however, engineers are more interesting in the global dynamical behaviors. It is the time for the biologists and engineers to work together for better understanding the complex networks.
\end{abstract}

\section{INTRODUCTION}

Nowadays complex networks are everywhere in our real life [1-14]. The ubiquity of networks attracts an increasing attention in various fields of science, especially in biological and engineering sciences. Biological networks are complex, with each individual typically closely related to all others, either directly or indirectly [2-10]. For example, an animate being is a very complex network. Also, many large engineering networks, such as Internet, World Wide Web and VLSI circuits, are very complex [11-14]. This mini review of the special session entitled "Complex Biological and Engineering Networks" in ISCAS 2007 provides a brief overview of the complex biological and engineering networks and bridges the gap between them.

The overall research goal of complex networks is to unravel the structure, function, dynamics and evolution of complex networks. Genetics and molecular biology normally uncovered the general principles for expressing genetic information. And genetics and biochemistry often identified some specific parts of individual protein networks. However, our understanding of complex networks as a whole is always limited, basically at a qualitative level. As E. O. Wilson said before [1], "The greatest challenge today, not just in cell biology and ecology but in all of science, is the accurate and complete description of complex systems. Scientists have broken down many kinds of systems. They think they know most of the elements and forces. The next task is to reassemble them, at least in mathematical models that capture the key properties of the entire ensembles."

Fortunately, more and more biologists realize the importance of uncovering the basic principles underlying the complex biological networks. There are some recent advances in this endeavor [7-9]: 1) Exploring the fundamental principles from the local rules of individual to the global behaviors of population; 2) Investigating the mechanisms from physiology to system dynamics; 3) Revealing the underlying rules from structure to dynamics in biological networks. For example, to simulate the collective motion of flocks and schools, several mathematical models are proposed recently, such as boid model [2], Vicsek model [3] and our model [5].

Similarly, there are some recent advances in understanding network structure and dynamics in complex engineering systems (consisting potentially of thousands and tens of thousands nodes). For example, the computerization of data acquisition and the availability of high computing power have led to the emergence of huge databases on various real networks of complex topology. Moreover, the public access to the huge amount of real data has in turn stimulated great interest in revealing the unifying properties of the different kinds of complex networks. To characterize the network structure, some typical models are then proposed over the past few decades, such as small-world and scale-free network models [1]. To understand the network dynamics, several specific dynamical network models are recently presented, such as the timevarying dynamical network model $[6,12]$.

The rest of this paper is organized as follows. In Section II, several evolving and dynamical models are introduced for modeling the complex biological networks and understanding the underlying biological mechanisms. Also, several evolving and dynamical models are proposed for designing and controlling complex engineering networks in Section III. And the conclusions and remarks are finally given in Section IV.

\section{Modeling Complex Biological Networks}

Biological networks are complex, such as a flock of birds as shown in Fig. 1 (a) and the food web of Little Rock Lake in Wisconsin [9] as shown in Fig. 1 (b), with each individual typically connected to all other individuals, either directly or indirectly. To model these biological networks, one introduces several evolving network models, including boid model [2], Vicsek model [3] and Couzin-Levin model [4], and a timevarying discrete network model in the following. 


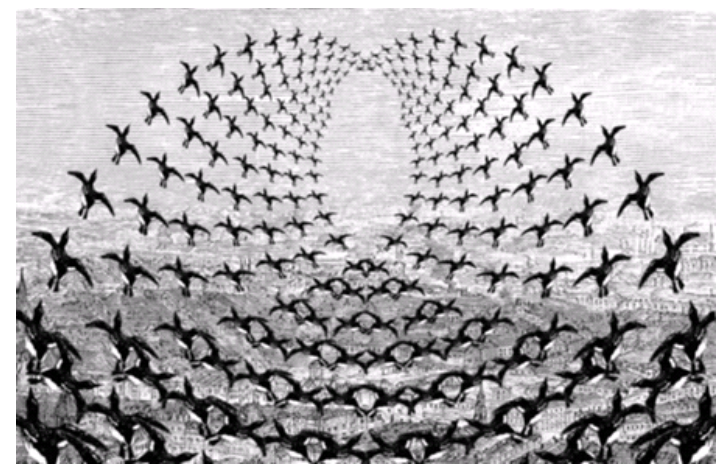

(a) A bird flock

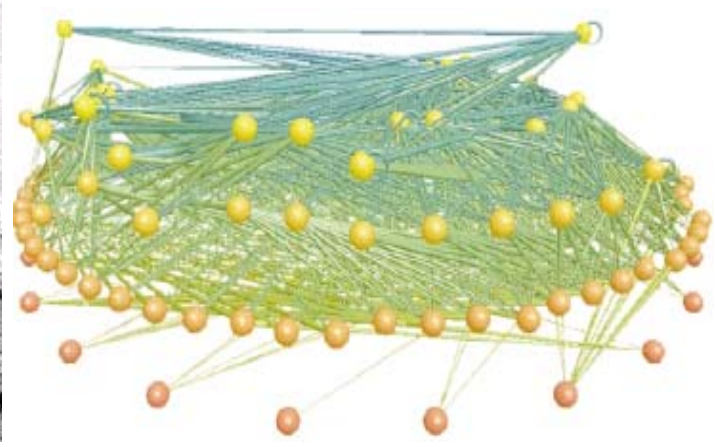

(b) Food web of Little Rock Lake, Wisconsin [9]

Fig. 1. Complex biological networks.

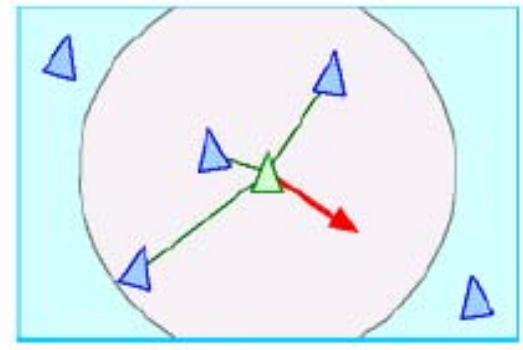

(a) Separation

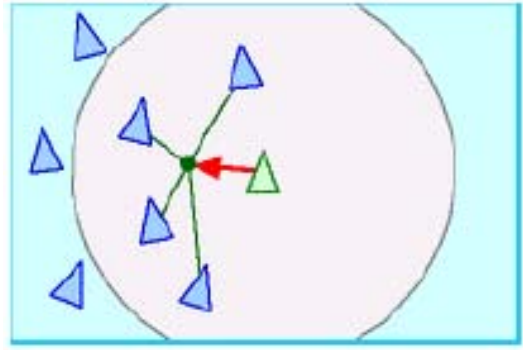

(b) Cohesion

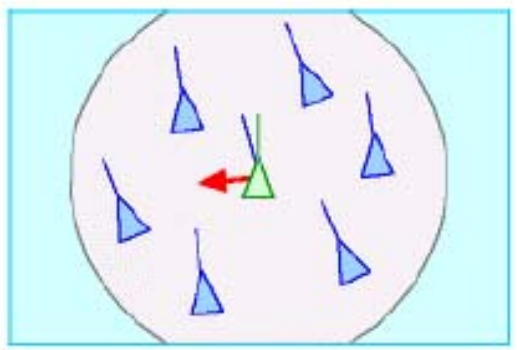

(c) Alignment

Fig. 2. The working principles of Boid model.

\section{A. Boid model}

We often watch a flock of birds flying to a specific direction or goal. There do not exist obvious leaders and individual birds move back and forth within the flock as shown in Fig. 1 (a). The motion of a flock of birds is one of nature's delights.

To simulate and understand the collective motion of bird flock, Reynolds [2] introduced a boid model in 1987, whose rules are described as follows:

(i) Separation: steer to avoid crowding local flockmates as shown in Fig. 2 (a).

(i) Cohesion: steer to move toward the average position of local flockmates as shown in Fig. 2 (b).

(ii) Alignment: steer towards the average heading of local flockmates as shown in Fig. 2 (c).

\section{B. Vicsek model}

To investigate the emergent mechanisms of self-ordered motion in systems of particles with biologically motivated interaction, Vicsek and his colleagues [3] proposed a simple model in 1995, called Vicsek model.

The updating rule of the position of the $i$ th individual is then given by

$$
\mathbf{x}_{i}(t+1)=\mathbf{x}_{i}(t)+\mathbf{v}_{i}(t) \Delta t,
$$

where $\mathbf{x}_{i}(t)$ is the position vector and $\mathbf{v}_{i}(t)$ is the velocity vector with the same absolute velocity $v$ and varying direction angle $\theta(t)$, which is updated by

$$
\theta_{i}(t+1)=<\theta(t)>_{r}+\Delta \theta,
$$

where $<\theta(t)>_{r}$ is the average direction angle of the particles (including particle $i$ ) being within a neighbor region of individual $i$ with radius $r$ and $\Delta \theta$ is a random noise with uniform distribution in the interval $\left[-\frac{\eta}{2}, \frac{\eta}{2}\right]$.

\section{Couzin-Levin model}

In 2005, Couzin and his colleagues [4] presented a simple model of collective motion. The updating rules are described as follows:

(i) If there exists $j$ satisfying $0<d_{i j} \leq \alpha$, then one has

$$
\mathbf{x}_{i}(t+\Delta t)=\sum_{0<d_{i j} \leq \alpha} \frac{\mathbf{x}_{i}(t)-\mathbf{x}_{j}(t)}{\left|\mathbf{x}_{i}(t)-\mathbf{x}_{j}(t)\right|},
$$

where $d_{i j}$ is the distance between individuals $i$ and $j, \Delta t$ is the time step, and $\alpha$ is the given minimum separation distance between individuals.

(ii) Otherwise, if there exists $j$ satisfying $\alpha<d_{i j} \leq \beta$, then one gets

$$
\mathbf{x}_{i}(t+\Delta t)=\sum_{\alpha<d_{i j} \leq \beta} \frac{\mathbf{x}_{j}(t)-\mathbf{x}_{i}(t)}{\left|\mathbf{x}_{j}(t)-\mathbf{x}_{i}(t)\right|}+\sum_{d_{i j} \leq \beta} \frac{\mathbf{v}_{j}(t)}{\left|\mathbf{v}_{j}(t)\right|},
$$

where $\beta$ is the given maximum reaction distance between individuals.

\section{A time-varying discrete biological network}

In real-world biological context, such as metapopulation, many different factors, such as ages, genders and species, 


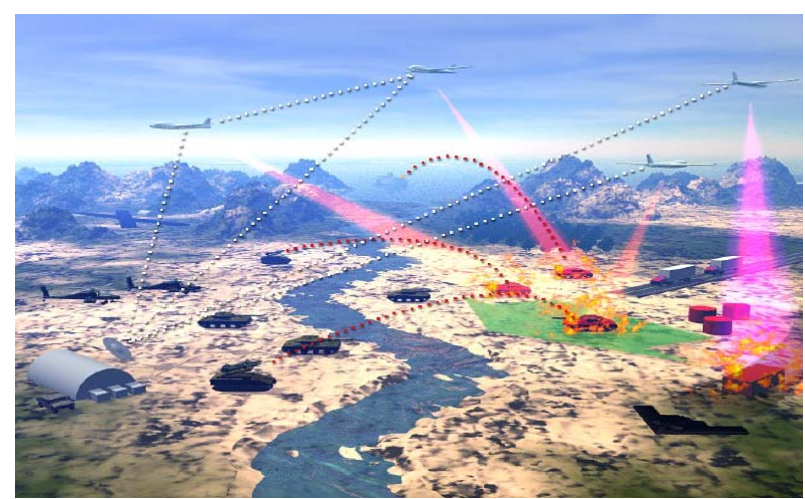

(a) Battlefield command network

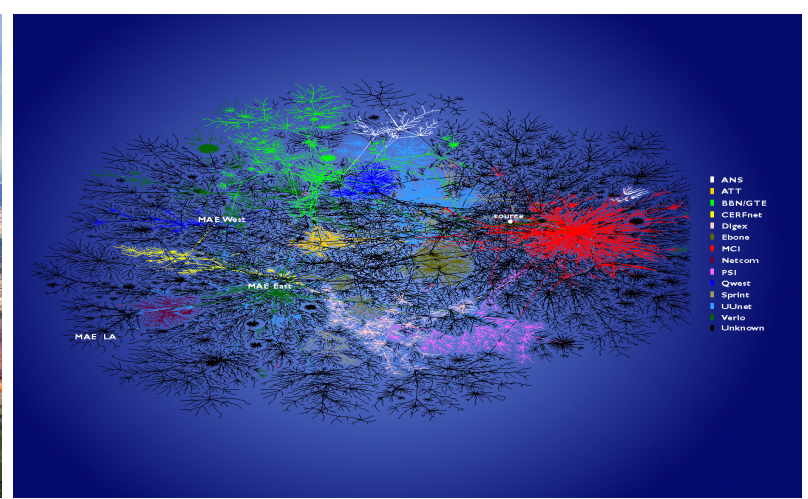

(b) World Wide Web

Fig. 3. Complex engineering networks.

are often involved simultaneously. The evolving rule of the population is then given by a multidimensional iterative map

$$
x(t+1)=f(x(t)),
$$

where $x \in \mathbb{R}^{n}$ and $f: X \rightarrow X\left(X \subseteq \mathbb{R}^{n}\right)$ is a fundamental reproductive (birth-death) function.

The time-varying discrete biological network with $N$ populations (habitat patches) is described by [6]

$$
x_{i}(t+1)=\sum_{j=1}^{N} c_{i j}(t) f\left(x_{j}(t)\right), i=1,2, \ldots, N,
$$

where $x_{i}(t) \in X$ is the patch density of the $i$ th habitat patch at time $t, \mathbf{C}(t)=\left(c_{i j}(t)\right)_{N \times N}$ is the dispersal matrix at time $t$, representing the coupling strength and the connecting status between patches.

In addition, $\mathbf{C}(t)$ satisfies

$$
\sum_{j=1}^{N} c_{i j}(t)=1 .
$$

That is, $\mathbf{C}(t)$ has row sums 1 for $t=1,2, \cdots$.

\section{Modeling Complex Engineering Networks}

Large engineering networks are also complex, such as Internet, large communication networks, power grid networks, Cellular Neural Networks, battlefield command networks as shown in Fig. 3 (a), and World Wide Web as shown in Fig. 3 (b).

\section{A. Small-world network}

To bridge the gap between a regular network and a random graph, Watts and Strogatz [1,14] introduced a small-world network model in 1998, which is given as follows:

(i) Initialization: Start with a nearest-neighbor coupled ring network with $N$ nodes, in which each node $i$ is connected to its $K$ neighboring nodes $i \pm 1, i \pm 2, \cdots, i \pm \frac{K}{2}$, where $K$ is an even integer. Also, suppose that $N \gg K \gg$ $\ln (N) \gg 1$, which makes the network be connected and sparse at all times. (ii) Randomization: Randomly reconnect each link of the network with probability $p$ satisfying that self-connections and duplicated links are excluded. Reconnecting means transferring one end of the connection to a randomly chosen node. This process introduces $\frac{p N K}{2}$ long-range links, which connect some nodes that otherwise would not have direct connections.

A small-world network lies along a continuum of network models between the two extreme networks: regular and random ones. After that, Newman and Watts $[1,14]$ modified the original WS model. Instead of rewiring links between nodes, extra links called shortcuts are added between pairs of nodes chosen at random in NW model, but no links are removed from the existing network. Therefore, the NW model reduces to the originally nearest-neighbor coupled network for $p=0$; while it becomes a globally coupled network for $p=1$. However, the NW model is equivalent to the WS model for sufficiently small $p$ and sufficiently large $N$ values.

\section{B. Scale-free Network}

Many real-world networks are scale-free. In 1999, Barabási and Albert [1,14] proposed a scale-free network model, which continuously evolves by the addition of new nodes and these new nodes are preferentially attached to existing nodes with large numbers of connections. The BA model is described as follows:

(i) Growth: Start with a small number $\left(m_{0}\right)$ of nodes, at each time step a new node is added and is connected to $\mathrm{m}\left(\leq m_{0}\right)$ already existing nodes.

(ii) Preferential attachment: When choosing the nodes to which the new node connects, assume that the probability $p_{i}$ that a new node will be connected to node $i$ depends on the degree $k_{i}$ of node $i$, satisfying

$$
p_{i}=\frac{k_{i}}{\sum_{j} k_{j}} .
$$

After $t$ time steps, this network has $N=t+m_{0}$ nodes and $m t$ links. According to the growth and preferential attachment, this network evolves into a scale-invariant state. That is, the 
probability of connecting a node with $k$ links is proportional to the power term $k^{-3}$.

\section{A time-varying discrete engineering network}

System (6) can be rewritten as follows [6]:

$$
x_{i}(t+1)=f\left(x_{i}(t)\right)+\sum_{j=1}^{N} \bar{c}_{i j}(t) f\left(x_{j}(t)\right), i=1,2, \ldots, N,
$$

where $\bar{c}_{i j}(t)=c_{i j}(t)$ for $i \neq j$ and $\bar{c}_{i i}(t)=c_{i i}(t)-1$ for $i=1,2, \ldots, N$. Then $\overline{\mathbf{C}}(t)=\mathbf{C}(t)-\mathbf{I}_{N \times N}$, where $\mathbf{I}_{N \times N}$ is an $N \times N$ unit matrix. Moreover, condition (7) is equivalent to

$$
\bar{c}_{i i}(t)=-\sum_{\substack{j=1 \\ j \neq i}}^{N} \bar{c}_{i j}(t), i=1,2, \ldots, N
$$

where $t=1,2, \cdots$. That is, $\overline{\mathbf{C}}(t)$ has zero row sums, which means that network (8) is a diffusive coupling network.

\section{A time-varying continuous engineering network}

Lü and his colleagues [12,14] introduced a general timevarying continuous engineering network

$$
\dot{x}_{i}(t)=f\left(x_{i}(t)\right)+\sum_{j=1}^{N} \bar{c}_{i j}(t) \mathbf{A}(t) x_{j}(t), i=1,2, \cdots, N,
$$

where $x_{i}(t)=\left(x_{i 1}(t), x_{i 2}(t), \cdots, x_{i n}(t)\right)^{T} \in \mathbb{R}^{n}$ is the state variable of node $i$ at time $t, \mathbf{A}(t)=\left(a_{k l}(t)\right)_{n \times n} \in$ $\mathbb{R}^{n \times n}$ is the inner-coupling matrix of the network at time $t$, $\overline{\mathbf{C}}(t)=\left(\bar{c}_{i j}(t)\right)_{N \times N}$ is the coupling configuration matrix representing the coupling strength and the connecting topology of the network at time $t$, in which $\bar{c}_{i j}(t)$ is defined as follows: if there is a connection from node $i$ to node $j(j \neq i)$ at time $t$, then $\bar{c}_{i j}(t) \neq 0$; otherwise, $\bar{c}_{i j}(t)=0(j \neq i)$, and the diagonal elements of matrix $\overline{\mathbf{C}}(t)$ satisfy the diffusively coupled conditions as follows:

$$
\bar{c}_{i i}(t)=-\sum_{\substack{j=1 \\ j \neq i}}^{N} \bar{c}_{i j}(t), \quad i=1,2, \cdots, N
$$

where $t \geq 0$

If $\mathbf{A}(t), \overline{\mathbf{C}}(t)$ are constant matrices, then network (10) becomes a time-invariant network [12-14]:

$$
\dot{x}_{i}(t)=f\left(x_{i}(t)\right)+\sum_{j=1}^{N} \bar{c}_{i j} \mathbf{A} x_{j}(t), i=1,2, \cdots, N .
$$

\section{CONCLUSIONS AND REMARKS}

We have briefly reviewed the main advances of the complex biological and engineering networks. These mathematical models belong to two different kinds of models: evolving model and dynamical model. Boid model, Vicsek model, Couzin-Levin model, small-world network, and scale-free network are the evolving models, which characterize the network structure and evolving mechanisms. The time-varying discrete biological network, the time-varying discrete and continuous engineering networks are the typical dynamical models, which characterize the network dynamics. These models provide some new insights for understanding the structures, functions, dynamics, and evolutions of complex biological and engineering networks. Different models focus on different aspects of complex networks. Although the biological and engineering networks are very different in the detailed contexts, they also have many similar network structures and dynamics.

Biologists pay more attention to the mechanics and local dynamics of individuals, however, engineers are more interesting in the global dynamical behaviors. It is the time for the biologists and engineers to work together for better understanding the complex biological and engineering networks now. This is the case not only in deeper and wider theoretical studies but also in many newly found real-world applied fields. It calls for the further efforts and endeavors from the communities of both biology and engineering, as well as the other close relative sciences.

\section{ACKNOWLEDGMENT}

This work was supported by the National Natural Science Foundation of China under Grants 60304017, 20336040, and 60221301, the Scientific Research Startup Special Foundation on Excellent PhD Thesis and Presidential Award of Chinese Academy of Sciences.

\section{REFERENCES}

[1] S. H. Strogatz, "Exploring complex networks," Nature, vol. 410, pp. 268-276, Mar. 2001.

[2] C. W. Reynolds, "Flocks, herds, and schools: a distributed behavioral model," Computer Graphics, vol. 21, no. 4, pp. 25-34, Jul. 1987.

[3] T. Vicsek, A. Czirók, E. Ben-Jacob, I. Cohen, and O. Shochet, "Novel type of phase transition in a system of self-driven particles," Phys. Rev. Lett., vol. 75, no. 6, pp. 1226-1229, Aug. 1995.

[4] I. D. Couzin, J. Krause, N. R. Franks, and S. A. Levin, "Effective leadership and decision-making in animal groups on the move," Nature, vol. 433, pp. 513-516, Feb. 2005.

[5] J. Lü, J. Liu, S. A. Simon, and I. D. Couzin, "Emerging consensus decision and robustness on the move of animal groups," preprint, 2006.

[6] L. Chen, J. Lü, J. Lu, and D. J. Hill, "Local asymptotic coherence of the time-varying discrete biological networks," preprint, 2006.

[7] J. M. Montoya, S. L. Pimm, and R. V. Solé, "Ecological networks and their fragility," Nature, vol. 442, pp. 259-264, Jul. 2006.

[8] N. Rooney, K. McCann, G. Gellner, and J. C. Moore, "Structural asymmetry and the stability of diverse food webs," Nature, vol. 442, pp. 265-269, Jul. 2006.

[9] M. Pascual, "Computational ecology: from the complex to the simple and back," PLoS Computational Biology, vol. 1, no. 2, pp. 0101-0105, Jul. 2005.

[10] R. V. Solé and S. Valverde, "Are network motifs the spandrels of cellular complexity," TRENDS in Ecology and Evolution, vol. 21, no. 8, pp. 419422, Aug. 2006.

[11] J. Zhou, J. Lu, and J. Lü, "Adaptive synchronization of an uncertain complex dynamical network," IEEE Trans. Auto. Contr., vol. 51, no. 4 , pp. 652-656, Apr. 2006.

[12] J. Lü and G. Chen, "A time-varying complex dynamical network model and its controlled synchronization criteria," IEEE Trans. Auto. Contr., vol. 50, no. 6, pp. 841-846, Jun. 2005.

[13] J. Lü, X. Yu, G. Chen, and D. Cheng, "Characterizing the synchronizability of small-world dynamical networks," IEEE Trans. Circuits Syst. I, vol. 51, no. 4, pp. 787-796, Apr. 2004.

[14] J. Lü, X. Yu, and G. Chen, "Chaos synchronization of general complex dynamical networks," Physica A, vol. 334, no. 1-2, pp. 281-302, Mar. 2004. 